\title{
Enhancement of Light Localization in Hybrid Thue-Morse/Periodic Photonic Crystals
}

\author{
Rihab Asmi, ${ }^{1}$ Naim Ben Ali, ${ }^{1,2}$ and Mounir Kanzari ${ }^{1}$ \\ ${ }^{1}$ Photovoltaic and Semiconductor Materials Laboratory, El-Manar University-ENIT, P.O. Box 37, Le Belvedere, \\ 1002 Tunis, Tunisia \\ ${ }^{2}$ College of Engineering, Industrial Engineering Department, Haïl University, Haïl 2440, Saudi Arabia
}

Correspondence should be addressed to Naim Ben Ali; naimgi2@yahoo.fr

Received 26 April 2016; Accepted 25 July 2016

Academic Editor: Francois M. Peeters

Copyright (C) 2016 Rihab Asmi et al. This is an open access article distributed under the Creative Commons Attribution License, which permits unrestricted use, distribution, and reproduction in any medium, provided the original work is properly cited.

The electric field intensity in one-dimensional (1D) quasiperiodic and hybrid photonics band-gap structures is studied in the present paper. The photonic structures are ordered according to Fibonacci, Thue-Morse, Cantor, Rudin-Shapiro, Period-Doubling, PaperFolding, and Baum-Sweet sequences. The study shows that the electric field intensity is higher for the Thue-Morse multilayer systems. After that the Thue-Morse structure will be combined with a periodic structure to form a hybrid photonic structure. It is shown that this hybrid system is the best for a strong localization of light. The proposed structures have been modeled using the Transfer Matrix Method.

\section{Introduction}

During the last decades, a great deal of attention has been devoted to photonic crystals (PC) as a new type of materials, whose optical properties are used to manipulate the light on the scale of the wavelength $[1,2]$. These crystals are structures whose dielectric index varies periodically on the scale of the wavelength on one, two, or three directions in space.

Although the two-dimensional (2D) and three-dimensional (3D) photonic crystals have attracted and still attract many research efforts, the one-dimensional structure (1D) is the simplest in both geometry and handling, allowing mastering the properties of these structures and studying the influence of various physical parameters on these properties.

It consists of a stack of alternating layers having low and high refractive indices, whose thicknesses satisfy the Bragg condition: $n_{L} d_{L}=n_{H} d_{H}=\lambda_{0} / 4$, where $\lambda_{0}$ is the reference wavelength. It is known as Bragg Mirror [3]. This Mirror produces light, which is propagating in the photonic crystal, a similar effect to that of the periodic potential of the electrons in a solid crystal. Just as in the case of a semiconductor, there appear forbidden bands for photons (PBGs) in photonic crystals prohibiting propagation of light in certain directions and for certain energies. The existence of PBGs will lead to many interesting phenomena.

During the last ten years, researchers have tried to fabricate several configurations of crystals to photonic band gap (PBG). Within this noteworthy structuring, light (or more generally an electromagnetic field) cannot propagate freely. It can be blocked (reflected), allowed only in certain directions, or even localized in certain areas [4].

Photonic crystals have paved the way for a new field of research and application possibilities. These crystals could for example improve the performance of lasers and lightemitting diodes or allow the manufacture of new types of antennas and amplifiers [5-7].

The concept of photonic crystal is not limited to periodic order. Since the discovery of quasiperiodic structures in 1984 by Shechtman et al. [8], most of the studies have concentrated on the localization properties of photons.

There is a wide variety of examples of one-dimensional quasicrystal [9]. The most common ones are Fibonacci, Thue-Morse (TM), Cantor, and Rudin-Shapiro structures. These structures have been widely studied, thus opening a way to a wide range of technological applications to many fields. 
The electronic properties of a one-dimensional quasicrystal arranged in a Fibonacci sequence, the wave functions at the center and at the edge of the band, the fractal nature (or self-similarity), and other critical properties of these wave functions are investigated by Kohmoto et al. [10].

Sibilia et al. [11] studied the self-similarity effects illustrated in the transmission spectrum of quasiperiodic structures arranged in a triadic Cantor.

Gellermann et al. [12] demonstrated experimentally the existence of band gaps in spectral response of these quasiperiodic structures which can be considered as evidence for the localization of the light waves.

Huang et al. [13] studied numerically the light localization of these structures by means of Transfer Matrix Method. They compare the localization length as a function of the chain length for the random, Thue-Morse, and Fibonacci sequences.

Vasconcelos et al. have studied, for the normal-incidence case, the electric field intensity in quasiperiodic structures: Fibonacci, Thue-Morse, and Period-Doubling [14]. Although Vasconcelos et al. have found that the electrical field distribution for each sequence follows its own structure, their study was not followed by an improvement of the intensity values (i.e., the improvement of the light localization). This is the concept of our study.

The aim of this work is to study and ameliorate the variation of the electric field intensity as a function of the thickness of quasiperiodic and hybrid photonics band-gap structures. These structures are composed of dielectric multilayer $H$ and $L$ stacked alternately following the Fibonacci, ThueMorse, Cantor, Rudin-Shapiro, Period-Doubling, PaperFolding, and Baum-Sweet sequences.

\section{Quasiperiodic Models}

2.1. The Fibonacci Sequence Case. The Fibonacci dielectric multilayer consists of two building blocks $H$ and $L$ with refraction indices $n_{H}$ (higher refractive index) and $n_{L}$ (lower refractive index) and thicknesses $d_{H}$ and $d_{L}$, respectively. The number of layers of the structure depends on the order of the Fibonacci sequence.

The general expression of this sequence is arranged according to the concatenation rule $S_{j+2}=S_{j}+S_{j+1}$ for $j \geq 0$, where $S_{j}$ is the $j$ th generation of Fibonacci structure. For example, $S_{2}=\{H L\}, S_{3}=\{L H L\}$, and $S_{4}=\{H L L H L\}$. Here, $S_{0}=\{H\}$ and $S_{1}=\{L\}[14]$.

2.2. The Thue-Morse Sequence Case. The Thue-Morse sequence can be also grown by juxtaposing the two building blocks $H$ and $L$ and can be produced by repeating application of the substitution rules $H \rightarrow H L$ and $L \rightarrow L H$. For example, the first few generations $S_{n}$ of Thue-Morse sequence are as follows: $S_{0}=\{H\}, S_{1}=\{H L\}, S_{2}=\{H L L H\}$, $S_{3}=\{H L L H L H H L\}$, and so on [15].

2.3. The Cantor Sequence Case. The distribution of Cantor consists in cutting each segment by three and the middle segment is removed. And the algorithm is repeated an infinite number of times.
Indeed, the Cantor sequence is created by the inflation rule: $H \rightarrow H L H$ and $L \rightarrow L L L$. The resulting set is the set of triadic Cantor $H \rightarrow H L H \rightarrow H L H L L L H L H \rightarrow$ HLHLLLHLHLLLLLLLLHLH, ...

2.4. The Rudin-Shapiro Sequence Case. The inflation rule used to generate the Rudin-Shapiro (RS) arrays can simply be obtained by the iteration of the two-letter inflation as follows: $H H \rightarrow H H H L, H L \rightarrow H H L H, L H \rightarrow L L H L$, and $L L \rightarrow$ LLLH.

2.5. The Period-Doubling Sequence Case. In a two-letter alphabet, the Period-Doubling sequence can simply be obtained by the substitution $H \rightarrow H L$ and $L \rightarrow H H$.

2.6. The Paper-Folding Sequence Case. This substitution rule is also simple, and like the other chains, if we start with the correct tile, the tiling is fixed: $H \rightarrow H H, H \rightarrow L H, L \rightarrow H L$, and $L \rightarrow L L$.

\section{Problem Formulation}

The method that we introduce here for calculating the electric field intensity spectra in one-dimensional photonic crystal is the Transfer Matrix Method (TMM) introduced by Yeh and Yariv [16]. This method is an excellent tool for accurately analyzing the electromagnetic wave propagation in stratified media which permits particularly extracting and solving the standard problem of the photonic band structures (transmission, reflection, and absorption) spectra [17]. In this method, the electromagnetic field is calculated gradually during the wave propagation in the structure.

To calculate the electric field intensity in a onedimensional multilayer system, it suffices to calculate the total field at the input of system $E_{\text {total }}(z=0)=E_{0,1}^{-}+E_{0}^{+}$ and add the field inside the system which varies depending on $z$ :

$$
E_{\text {total }}(z)=E_{j-1, j}^{+}+E_{j-1, j}^{-}
$$

$E_{0,1}^{-}$is the reflected wave by the whole system, $E_{0}^{+}$is the incident wave.

The Transfer Matrix Method related the amplitudes of the incident wave $E_{0}^{+}$, reflected wave $E_{0,1}^{-}$, and transmitted wave $E_{m, m+1}^{+}$(Figure 1). This is expressed by the following matrix relation:

$$
\begin{aligned}
\left(\begin{array}{c}
E_{j-1, j}^{+} \\
E_{j-1, j}^{-}(z)
\end{array}\right)= & C_{2,1} C_{3,2} \cdots C_{m+1, m} \\
& \times\left(\begin{array}{c}
E_{0}^{+} \\
E_{\text {total }}(z=0)
\end{array}\right) \\
C_{2,1} C_{3,2} \cdots C_{m+1, m}= & \left(\begin{array}{ll}
a & b \\
c & d
\end{array}\right) .
\end{aligned}
$$




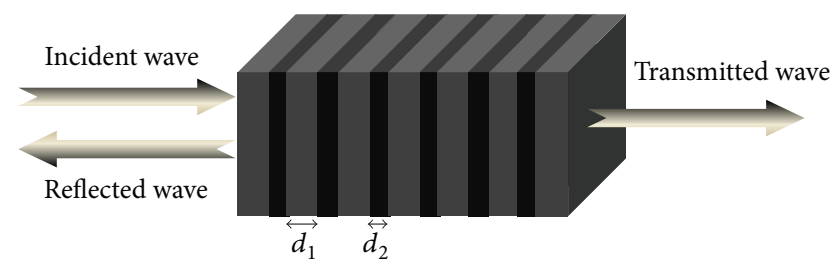

FIGURE 1: 1D periodic photonic crystal composed of alternating layers of dielectric permittivities $\varepsilon_{1}$ and $\varepsilon_{2}$ and thicknesses $d_{1}$ and $d_{2}$.

$C_{j-1, j}$ is the transfer matrix which is the product of a transition matrix $C_{\mathrm{ps}}$ and a propagation matrix $C_{\mathrm{pr}}$ :

$$
\begin{aligned}
C_{j-1, j}=C_{\mathrm{pr}} \cdot C_{\mathrm{ps}} \\
=\frac{1}{t_{j, j-1}} \\
\quad \times\left(\begin{array}{cc}
\exp \left(-i \phi_{j-1}\right) & -r_{j-1, j} \exp \left(i \phi_{j-1}\right) \\
-r_{j-1, j} \exp \left(-i \phi_{j-1}\right) & \exp \left(i \phi_{j-1}\right)
\end{array}\right) .
\end{aligned}
$$

$t_{j}$ and $r_{j}$ are the Fresnel transmission and reflection coefficients between the layers $(j-1)$ and $j$ :

$$
\begin{aligned}
r_{j, j-1} & =\frac{n_{j} \cos \theta_{j}-n_{j-1} \cos \theta_{j-1}}{n_{j} \cos \theta_{j}+n_{j-1} \cos \theta_{j-1}}=-r_{j-1, j}, \\
t_{j, j-1} & =\frac{2 n_{j} \cos \theta_{j}}{n_{j} \cos \theta_{j}+n_{j-1} \cos \theta_{j-1}} .
\end{aligned}
$$

The values $\varphi_{j-1}$ that appear in the expression of the propagation matrix indicate the phase changes between the interfaces $(j-1)$ and $j$ and are expressed by

$$
\varphi_{j-1}=\frac{2 \pi}{\lambda} n_{j-1} d_{j-1} \cos \left(\theta_{j-1}\right), \quad \varphi_{0}=0,
$$

where $\lambda$ is the wavelength of the incident wave over the structure and $d_{j-1}$ is the thickness of the $(j-1)$ th layer. The complex refractive indices $N_{j}=n_{j}+i k_{j}$ and the complex angles of incidence $\theta_{j}$ obviously follow Snell's law:

$$
N_{j-1} \sin \theta_{j-1}=N_{j} \sin \theta_{j},
$$

with $j=[1, m+1]$.

These equations allow deducing the expression of the total electric field according to the thickness $(z)$ :

$$
E_{\text {total }}(z)=E_{j-1, j}^{+}(z)+E_{j-1, j}^{-}(z) .
$$

Finally, the intensity of the total electric field will be

$$
I=\left|E_{\text {total }}(z)\right|^{2}
$$

\section{Results and Discussions}

4.1. Quasiperiodic Systems. In this numerical investigation, silicon dioxide $\mathrm{SiO}_{2}(L)$ and titanium dioxide $\mathrm{TiO}_{2}(H)$ are chosen as two elementary layers, with refractive indices $n_{L}=$ 1.45 and $n_{H}=2.3$, respectively. The thicknesses of both kinds of layers in different structures are $d_{L}=\lambda_{0} / 4 n_{L}$ and $d_{H}=\lambda_{0} / 4 n_{H}$ (Bragg condition), with $\lambda_{0}=0.5 \mu \mathrm{m}$ as the reference wavelength. For our study, all materials are assumed to be linear, homogeneous, and nonabsorbing. We assume that the front and the back media have refractive index $n_{0}=1$ (air refractive index).

We propose here showing the influence of optogeometrical parameters of $1 \mathrm{D}$ photonic structure on the localization of the electromagnetic field. We compare the distributions of Cantor, Fibonacci, Thue-Morse, Rudin-Shapiro, PaperFolding, Period-Doubling, and Baum-Sweet for an almost equal number of layers.

The strong localization of the electromagnetic field in the material requires structures in which the electromagnetic wave is slowed sharply to certain frequencies. From Figure 2, it is clear that the Thue-Morse distribution is the best structure to localize light; it permits getting the highest electric field intensity $\left(2,5 \cdot 10^{5}\right.$ u.a) with a number of layers equal to 128 . Here and in contrast to other structures, the wave is located at the outlet of the structure.

4.2. Hybrid Systems. In this second part, we will study the variation of the electric field intensity as a function of the thickness of one-dimensional hybrid systems. The hybrid assembly design implies putting the periodic and the quasiperiodic multilayer together in a sandwich configuration. The quasiperiodic photonic crystals (PCs) used in these hybrid structures are the Thue-Morse sequence, the best performing structure that we have found in the first part. The geometrical configurations considered are of the types Bragg/Thue-Morse/Bragg and ThueMorse/Bragg/Thue-Morse.

4.2.1. The Hybrid $H(L H)_{j} /(T M)_{n} / H(L H)_{j}$ Systems. In the first section, we are interested in the multilayer system composed of Thue-Morse sequence $(\mathrm{TM})_{n}$ sandwiched between two periodic multilayer systems $H(L H)_{j}$ (Bragg mirror) (Figure 3 ), where $(n)$ and $(j)$ are the number of iterations of the Thue-Morse and the periodic systems, respectively.

(1) The Optimization of the Repetition Number $j$ of the Periodic System. In order to optimize the repetition number $j$ of the periodic multilayer structure $H(L H)_{j}$, we fix the iteration number of the Thue-Morse $n$ to 3 and let $j$ vary from 1 to 50. Figure 4 shows the variation of the maximal electric field intensity for different values of $j$. From Figure 4, we notice that when $j$ is equal to 25 we obtain the highest intensity. By moving away from this value, a decrease of the maximal value of intensity is noticed. Then, we can conclude that the optimal value of $j$ which can be chosen for the configuration $H(L H)_{j} /(\mathrm{TM})_{3} / H(L H)_{j}$ is equal to 25 .

(2) Variation Effect of the Repetitive Number $n$. Now we move to study the electric field intensity of the configuration $H(L H)_{25} /(\mathrm{TM})_{n} / H(L H)_{25}$ for the optimal value $j=25$ and for different values of $n$ ranges from 1 to 11. From Figure 5, we can conclude that the electric field intensity increases with 


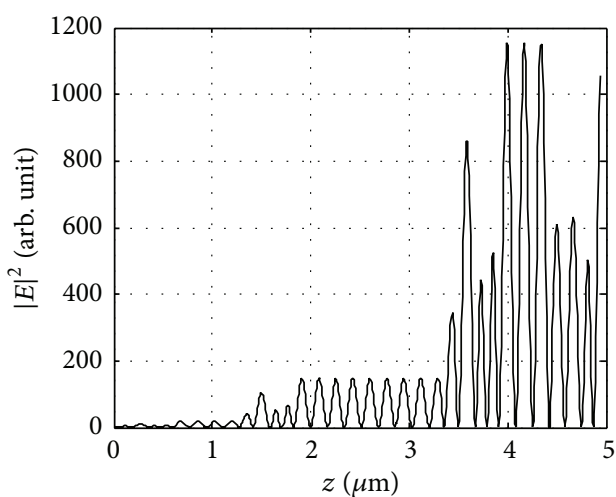

(a)

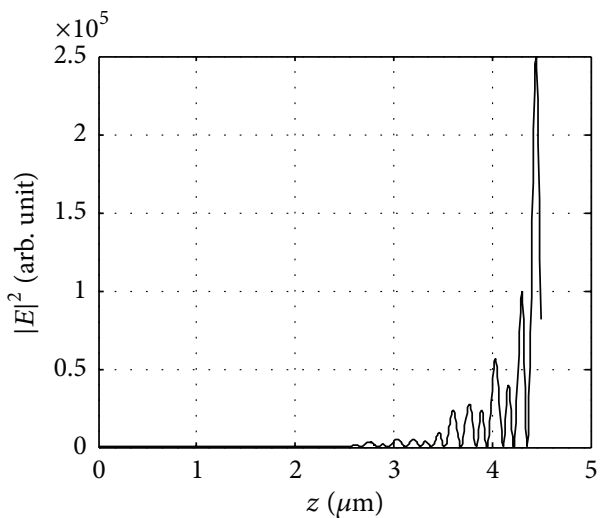

(c)

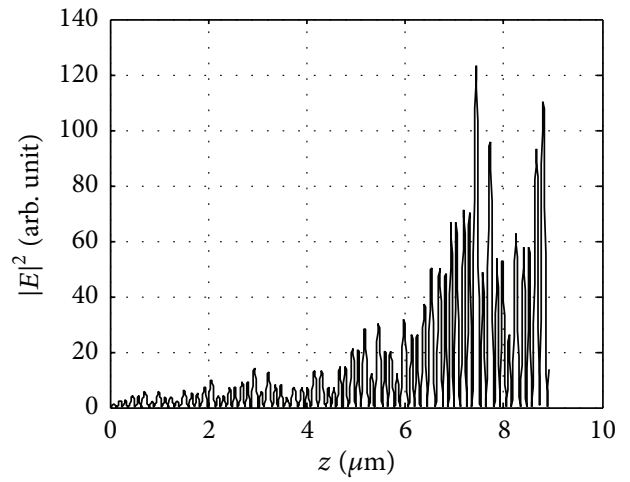

(e)

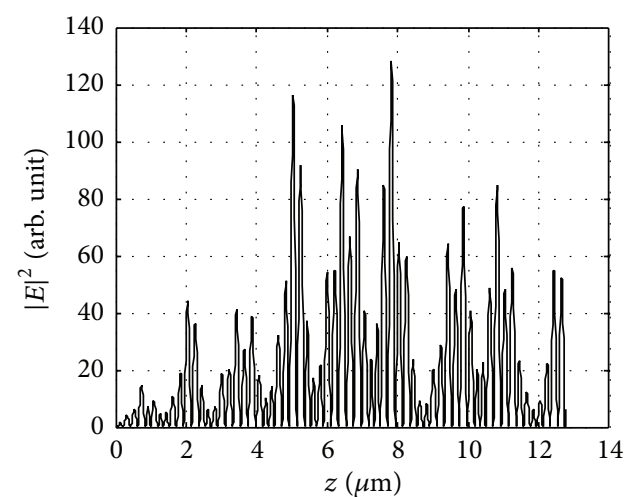

(b)

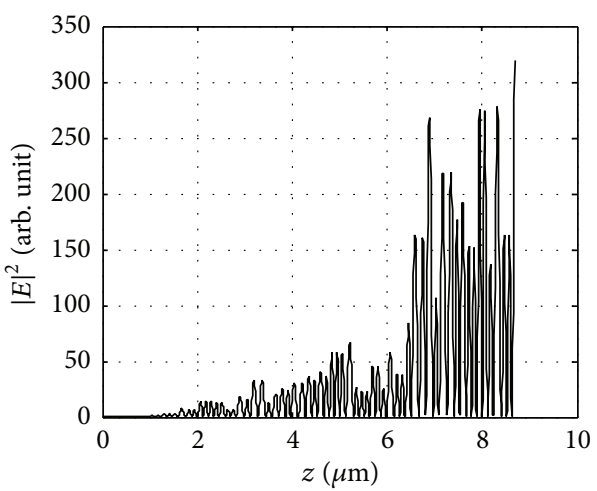

(d)

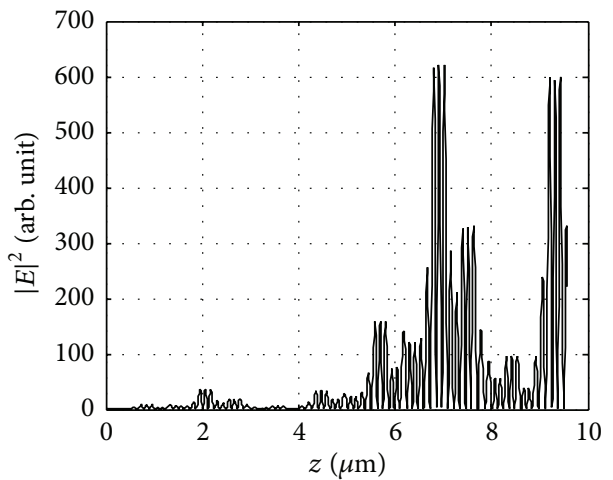

(f)

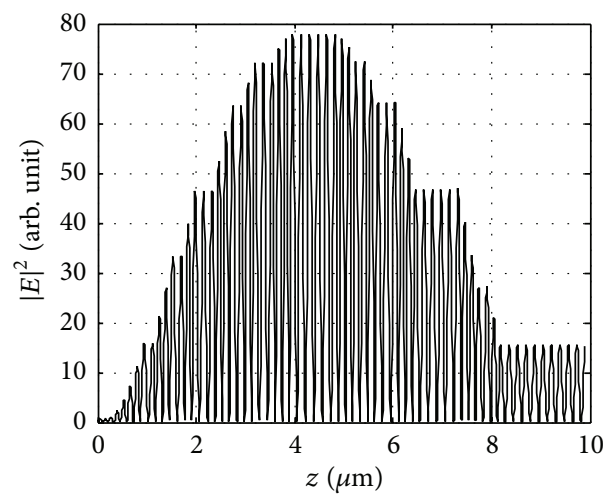

(g)

FiguRE 2: Electric field intensity of quasiperiodic photonic crystals according to the thickness ( $z$ ): (a) Cantor sequence (81 layers), (b) Fibonacci sequence (89 layers), (c) Thue-Morse sequence (128 layers), (d) Rudin-Shapiro sequence (128 layers), (e) Paper-Folding (128 layers), (f) Period-Doubling (128 layers), and (g) Baum-Sweet (128 layers). 


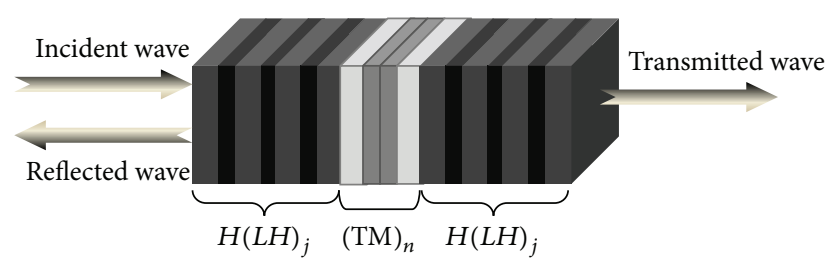

Figure 3: Geometry example of the hybrid system formed by a Thue-Morse sequence sandwiched between two periodic structures.

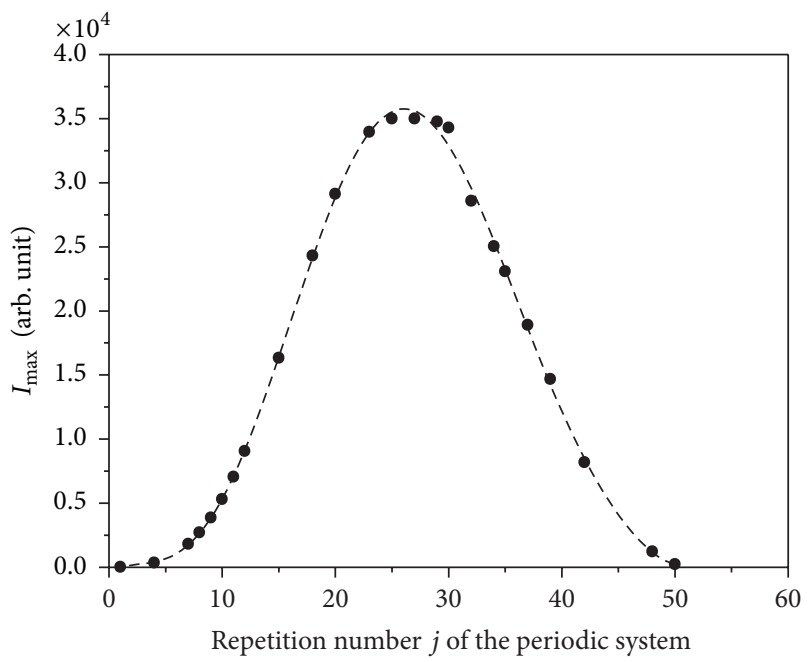

FIGURE 4: Variation of the maximal electric field intensity according to the parameter $j$ and for the $H(L H)_{j} /(\mathrm{TM})_{3} / H(L H)_{j}$ system.

the parameter $n$. For the values $n \geq 4$, the intensity increases rapidly. If $n=4$, the total length of the hybrid structure is $p=118$ layers. This value $(n=4)$ is useful if we seek to experimentally achieve the hybrid structure because if $n>4$ the total number of layers grows rapidly and the structure becomes experimentally infeasible. For example, if $n=11$ the intensity value exceeds $6 \cdot 10^{6}$ u.a but the total number of layers becomes 2150 (Figure 5). Consequently, we choose the value of $n=4$ which corresponds to acceptable system performances with minimal values of layers.

4.2.2. The Hybrid $(T M)_{n} / H(L H)_{j} /(T M)_{n}$ Systems. In the second section, the spectra of intensity variation were obtained at normal incidence for a hybrid structure composed of a periodic photonic crystal interposed between two Thue-Morse sequences $(\mathrm{TM})_{n} / H(L H)_{j} /(\mathrm{TM})_{n}$ (see Figure 6), where $(n)$ and $(j)$ are the number of iterations of the ThueMorse and the periodic systems, respectively.

(1) The Optimization of the Repetition Number $j$ of the Periodic System. To determine the optimal value of $j$ of the periodic system $H(L H)_{j}$, we study the optical response of the hybrid $(\mathrm{TM})_{n} / H(L H)_{j} /(\mathrm{TM})_{n}$ system for different values of $j$ and by fixing $n$ at 3 . The variation of the maximal electric field intensity showed in Figure 7 indicates that the optimal value of $j$ for the configuration $(\mathrm{TM})_{3} / H(L H)_{j} /(\mathrm{TM})_{3}$ is

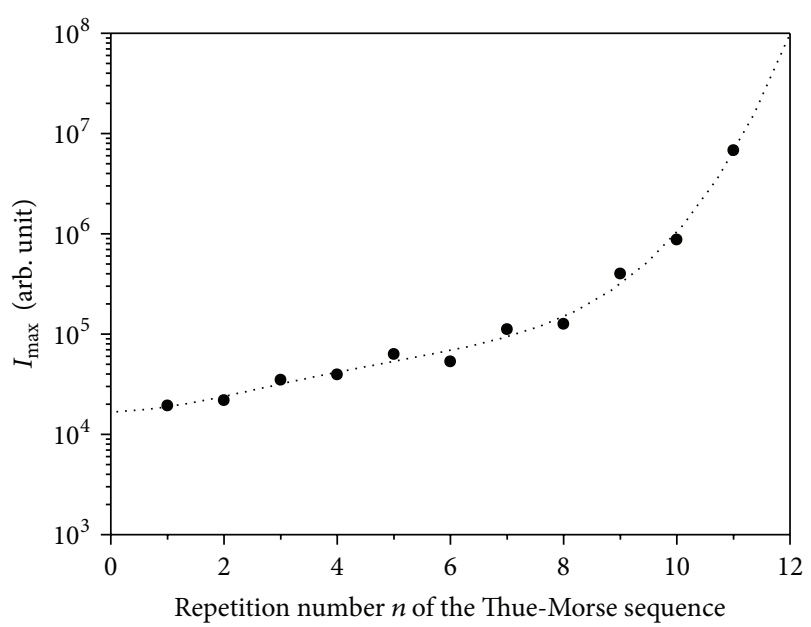

FIGURE 5: Variation of the maximal electric field intensity according to the parameter $n$ and for the $H(L H)_{25} /(\mathrm{TM})_{n} / H(L H)_{25}$ system.

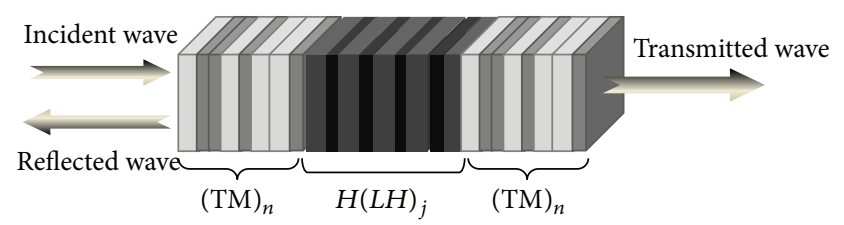

FIgURE 6: Geometry example of the hybrid system formed by a periodic sequence sandwiched between two Thue-Morse sequences.

26 corresponding to the highest value of the electric field intensity.

(2) Variation Effect of the Repetitive Number $n$. The optimal value $j=26$ is chosen and the variation of the parameter $n$ is studied for the configuration $H(L H)_{26} /(\mathrm{TM})_{n} / H(L H)_{26}$. Figure 8 shows the variation of the maximal electric field intensity inside the hybrid photonic structure as function of the parameter $n$. The values of the maximal electric field intensity are governed approximately by a simple refined function according to $n$ by

$$
I_{\max }=345.4 * n+100 .
$$

As in the previous section and to optimize the total number of layers of the hybrid structure, the value $n=4$ is chosen.

Figures 5 and 8 show that the structure $H(L H)_{j} /$ $(\mathrm{TM})_{n} / H(L H)_{j}$ is better than the structure $(\mathrm{TM})_{n} / H(L H)_{j} /$ $(\mathrm{TM})_{n}$ for the localization of light and the optimization of the total number of layers (i.e., optimization of the hybrid photonic system thickness).

\section{Conclusions}

In this paper we have studied firstly the localization of light in one-dimensional quasiperiodic photonic crystals, according to Fibonacci, Thue-Morse, Cantor, Rudin-Shapiro, PeriodDoubling, Paper-Folding, and Baum-Sweet sequences.

By comparing these structures, we try to highlight the importance of light localization in quasiperiodic photonic 


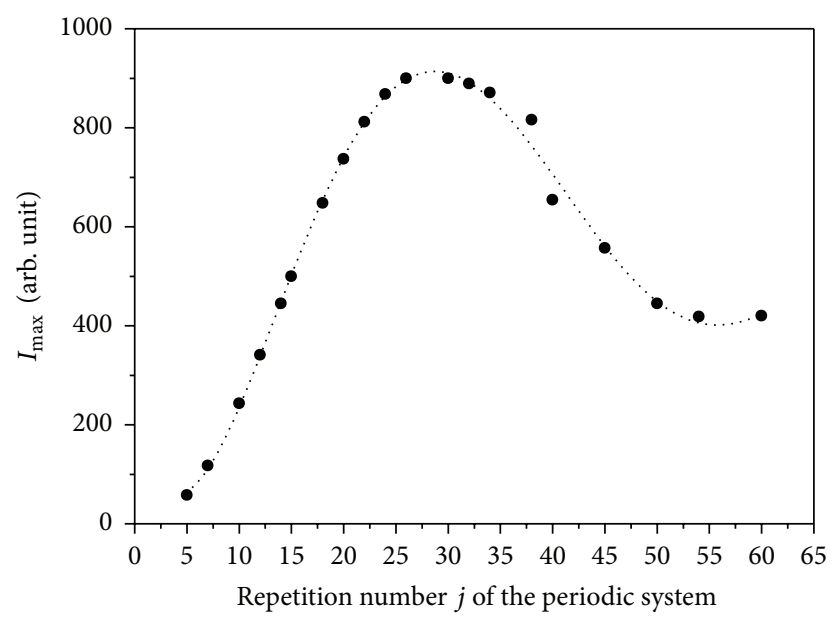

FIGURE 7: Variation of the maximal electric field intensity according to the parameter $j$ and for the $(\mathrm{TM})_{3} / H(L H)_{j} /(\mathrm{TM})_{3}$ system.

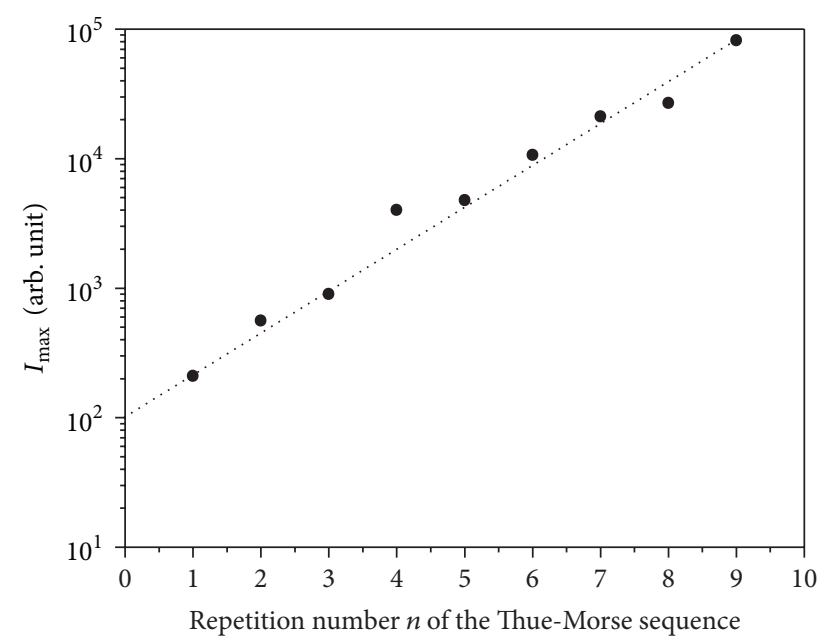

FIGURE 8: Variation of the maximal electric field intensity according to the parameter $n$ and for the $(\mathrm{TM})_{n} / H(L H)_{26} /(\mathrm{TM})_{n}$ system.

crystals. We found that the Thue-Morse distribution is the best structure to localize light; it permits getting the highest electric field intensity.

Secondly, to enhance the importance of this structure and to emphasize the importance of hybrid system, we have made an optimization by studying the combination of Thue-Morse and periodic structures.

We found that the hybrid photonic system (Bragg/ThueMorse/Bragg) is performing well in localizing light, as well as optimizing the number of layers and the thickness of the photonic system.

\section{Competing Interests}

The authors certify that they have no affiliations with or involvement in any organization or entity with any financial interest (such as honoraria; educational grants; participation in speakers' bureaus; membership, employment, consultancies, stock ownership, or other equity interest; and expert testimony or patent-licensing arrangements) or nonfinancial interest (such as personal or professional relationships, affiliations, knowledge or beliefs) in the subject matter or materials discussed in this paper. The paper represents valid work; neither this paper nor one with substantially similar content under our authorship has been published or is being considered for publication elsewhere; and copies of any closely related manuscripts are enclosed in the paper submission.

\section{References}

[1] J. D. Joannopoulos, P. R. Villeneuve, and S. Fan, "Photonic crystals: putting a new twist on light," Nature, vol. 386, pp. 143149, 1997.

[2] X.-Y. Wu, B.-J. Zhang, X.-J. Liu et al., "Light field distribution of general function photonic crystals," Physica E: LowDimensional Systems and Nanostructures, vol. 46, pp. 133-138, 2012.

[3] P. Yeh, Optical Waves in Layered Media, Wiley Series in Pure and Applied Optics, John Wiley \& Sons, New York, NY, USA, 1988.

[4] A. Lagendijk, G. J. Rivas, A. Imhof, F. J. P. Schuurmans, and R. Sprik, "Propagation of light in disordered semiconductors," in Proceedings of the NATO ASI 'Photonic Crystals and Light Localization in the 21st century', pp. 447-473, Kluwer, Dordrecht, Netherlands, 2001.

[5] X. Li, K. Xie, and H.-M. Jiang, "Properties of defect modes in one-dimensional photonic crystals containing two nonlinear defects," Optics Communications, vol. 282, no. 21, pp. 42924295, 2009.

[6] K. Jamshidi-Ghaleh and Z. Safari, "Effect of a subwavelength layer on all optical-diode action in 1D photonic crystal," International Journal of Modern Physics: Conference Series, vol. 15, pp. 48-53, 2012.

[7] S. J. Dewhurst, D. Granados, D. J. P. Ellis et al., "Slow-lightenhanced single quantum dot emission in a unidirectional photonic crystal waveguide," Applied Physics Letters, vol. 96, no. 3, Article ID 031109, 2010.

[8] D. Shechtman, I. Blech, D. Gratias, and J. W. Cahn, "Metallic phase with long-range orientational order and no translational symmetry," Physical Review Letters, vol. 53, no. 20, pp. 1951-1953, 1984.

[9] E. Maciá, “The role of aperiodic order in science and technology," Reports on Progress in Physics, vol. 69, no. 2, pp. 397-441, 2006.

[10] M. Kohmoto, B. Sutherland, and K. Iguchi, "Localization of optics: quasiperiodic media," Physical Review Letters, vol. 58, no. 23, pp. 2436-2438, 1987.

[11] C. Sibilia, P. Masciulli, and M. Bertolotti, "Optical properties of quasiperiodic (self-similar) structures," Pure and Applied Optics, vol. 7, no. 2, pp. 383-391, 1998.

[12] W. Gellermann, M. Kohmoto, B. Sutherland, and P. C. Taylor, "Localization of light waves in Fibonacci dielectric multilayers," Physical Review Letters, vol. 72, no. 5, pp. 633-636, 1994.

[13] D. Huang, G. Gumbs, and M. Kolár, "Localization in a onedimensional Thue-Morse chain," Physical Review B, vol. 46, pp. 11479-11486, 1992. 
[14] M. S. Vasconcelos, E. L. Albuquerque, and A. M. Mariz, "Optical localization in quasi-periodic multilayers," Journal of Physics: Condensed Matter, vol. 10, no. 26, pp. 5839-5849, 1998.

[15] M. Kolar, M. K. Ali, and F. Nori, "Generalized Thue-Morse chains and their physical properties," Physical Review B, vol. 43, no. 1, pp. 1034-1047, 1991.

[16] P. Yeh and A. Yariv, Optical Waves in Crystalsed, vol. 589 of Wiley Series in Pure and Applied Optics, 1984.

[17] D. Levine and P. J. Steinhardt, "Quasicrystals: a new class of ordered structures," Physical Review Letters, vol. 53, no. 26, pp. 2477-2480, 1984. 

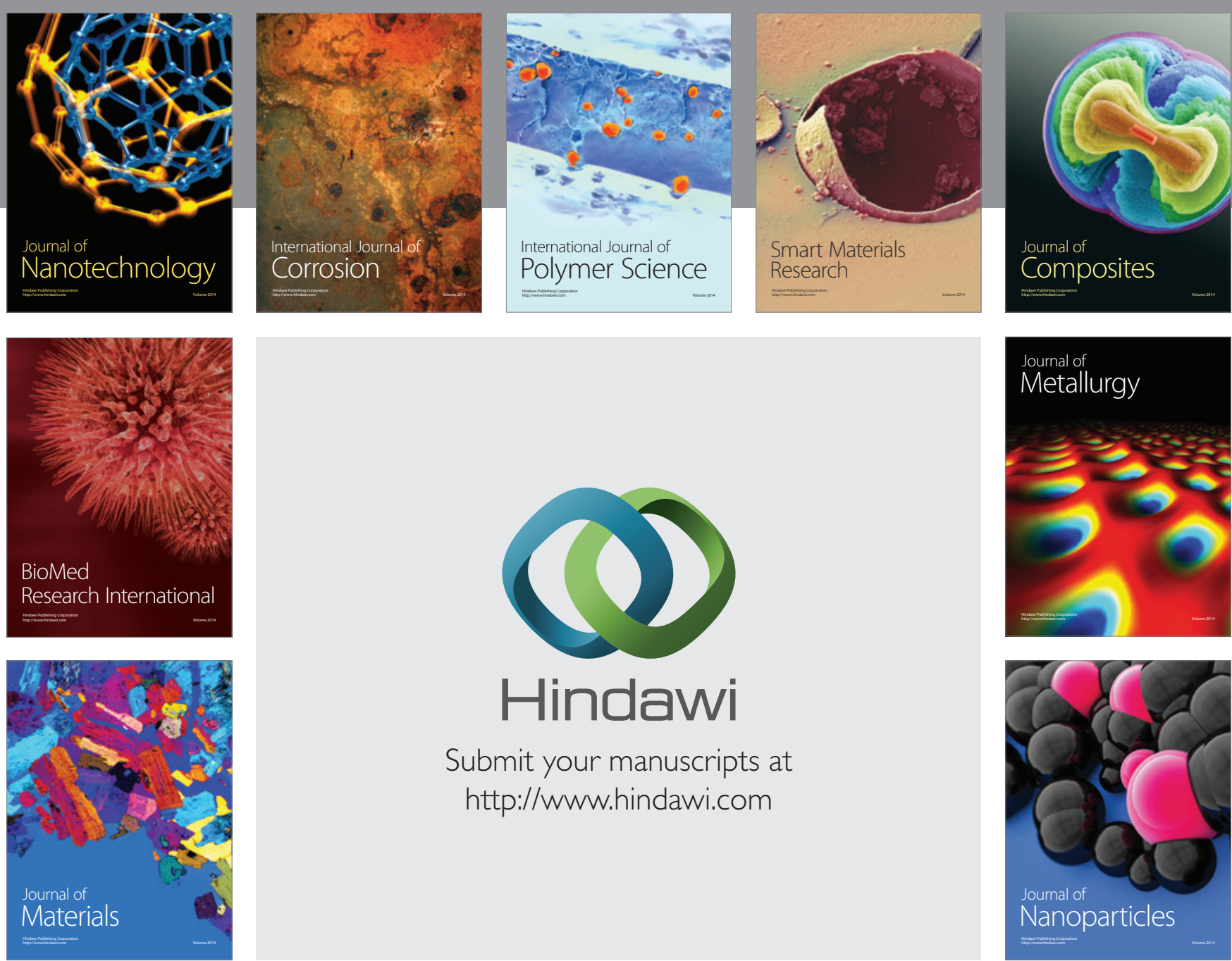

\section{Hindawi}

Submit your manuscripts at

http://www.hindawi.com

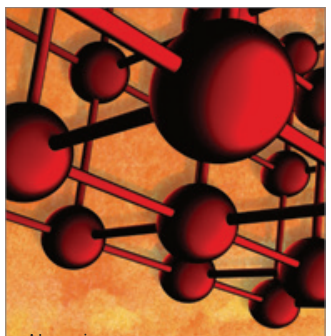

Materials Science and Engineering
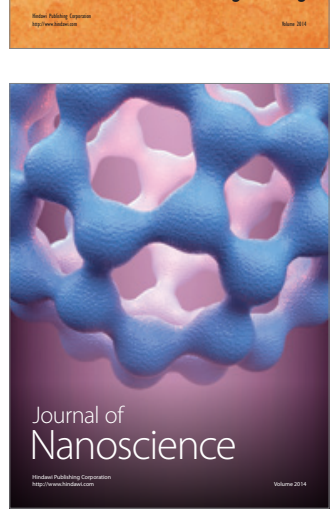
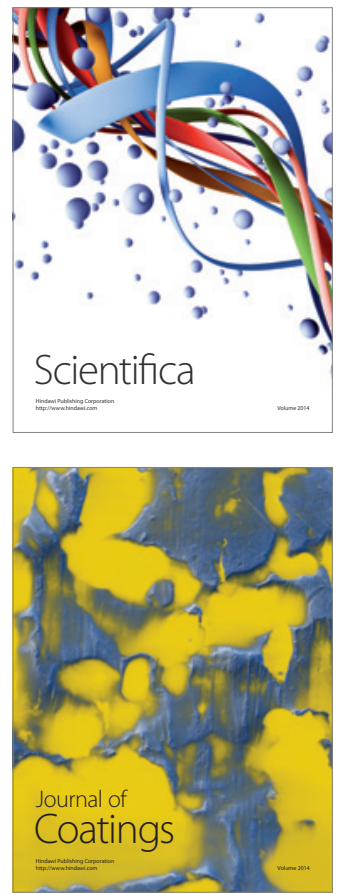
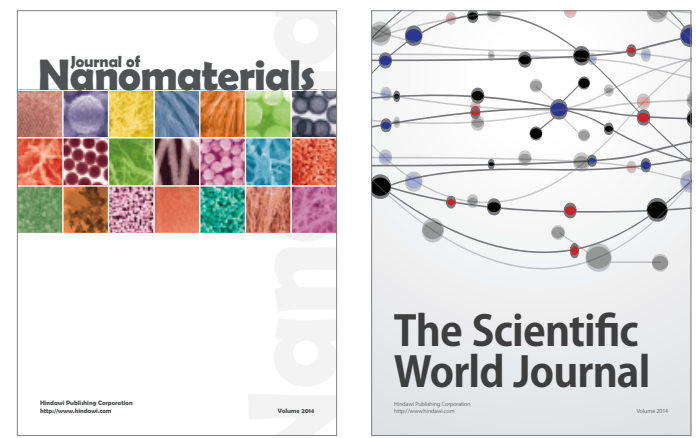

The Scientific World Journal
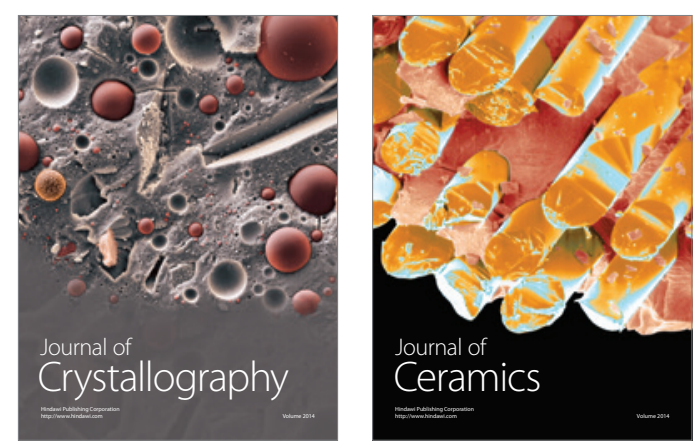
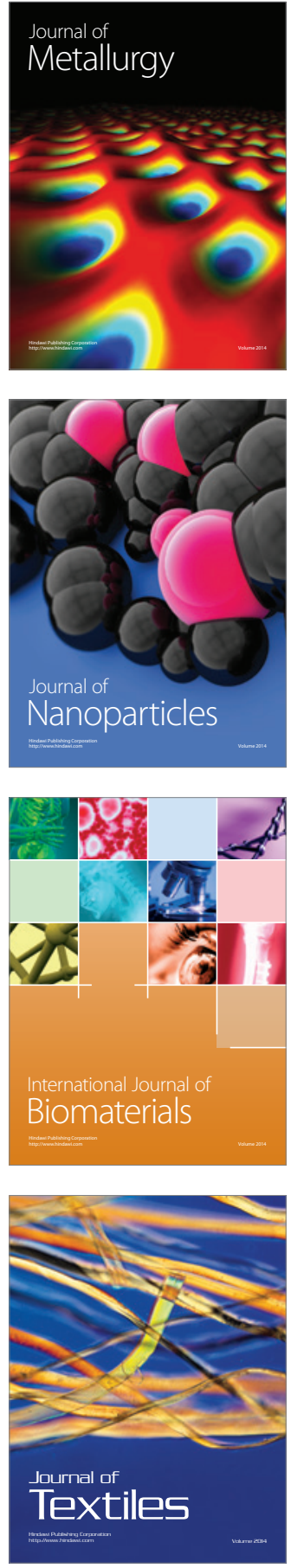